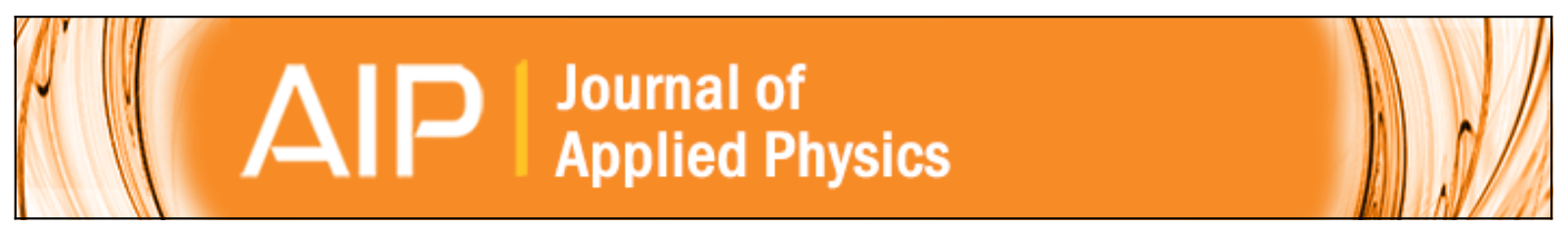

\title{
Enhanced thermoelectric power in ultrathin topological insulators with magnetic doping
}

M. Tahir, A. Manchon, and U. Schwingenschlögl

Citation: Journal of Applied Physics 116, 093708 (2014); doi: 10.1063/1.4894283

View online: http://dx.doi.org/10.1063/1.4894283

View Table of Contents: http://scitation.aip.org/content/aip/journal/jap/116/9?ver=pdfcov

Published by the AIP Publishing

\section{Articles you may be interested in}

Enhanced thermoelectric performance of nanostructured topological insulator $\mathrm{Bi} 2 \mathrm{Se} 3$

Appl. Phys. Lett. 106, 053102 (2015); 10.1063/1.4907252

Inhomogeneous thermal conductivity enhances thermoelectric cooling

AlP Advances 4, 124501 (2014); 10.1063/1.4903547

The maximal cooling power of magnetic and thermoelectric refrigerators with $\mathrm{La}(\mathrm{FeCoSi}) 13$ alloys

J. Appl. Phys. 113, 17A945 (2013); 10.1063/1.4801424

Magnetically doped semiconducting topological insulators

J. Appl. Phys. 112, 063912 (2012); 10.1063/1.4754452

Thermoelectric efficiency of topological insulators in a magnetic field

J. Appl. Phys. 111, $07 E 319$ (2012); 10.1063/1.3672847

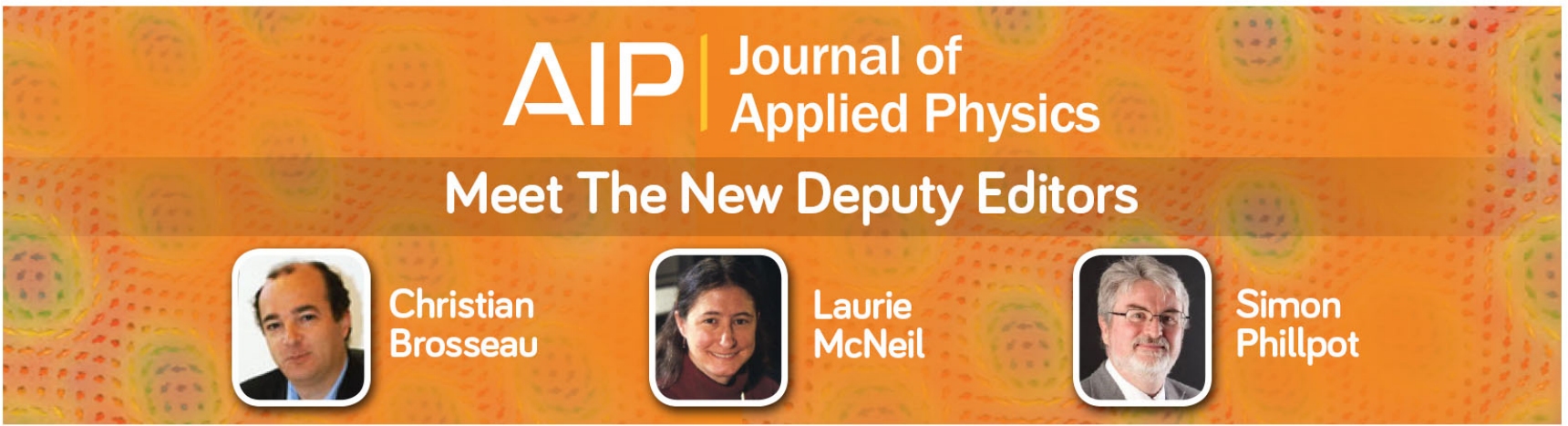




\title{
Enhanced thermoelectric power in ultrathin topological insulators with magnetic doping
}

\author{
M. Tahir, A. Manchon, and U. Schwingenschlögla \\ PSE Division, KAUST, Thuwal 23955-6900, Kingdom of Saudi Arabia
}

(Received 10 July 2014; accepted 19 August 2014; published online 4 September 2014)

\begin{abstract}
We derive analytical expressions for the magnetic moment and orbital magnetization as well as for the corresponding thermal conductivity and thermoelectric power of a topological insulator film. We demonstrate enhancement of the thermoelectric transport for decreasing film thickness and for application of an exchange field due to the tunable band gap. Combining hybridization and exchange field is particularly suitable for heat to electric energy conversion and thermoelectric cooling. (C) 2014 AIP Publishing LLC. [http://dx.doi.org/10.1063/1.4894283]
\end{abstract}

\section{INTRODUCTION}

Three-dimensional topological insulators, such as $\mathrm{Bi}_{2} \mathrm{Se}_{3}$ and $\mathrm{Bi}_{2} \mathrm{Te}_{3}$, exhibit a linear dispersion of the surface states in the bulk band gap. ${ }^{1-3}$ The gapless surface states are robust against perturbations that maintain the time-reversal symmetry and offer a unique platform to realize various exotic phenomena. ${ }^{4,5}$ Breaking of the time-reversal symmetry by magnetic doping ${ }^{6,7}$ leads to insulating surface states with an anomalous Hall effect. ${ }^{8}$ A band gap in the surface states can also occur in ultrathin films of three dimensional nonmagnetic topological insulators. For film thicknesses of $5 \mathrm{~nm}$ or less, the quantum tunneling between the upper and lower surfaces becomes non-negligible, inducing a hybridization gap at the Dirac point. Thin films of $\mathrm{Bi}_{2} \mathrm{Se}_{3}$ have been fabricated by the molecular beam epitaxy technique, ${ }^{3,9}$ and an insulating behavior in $\mathrm{Bi}_{2} \mathrm{Se}_{3}$ field effect transistors has been demonstrated recently. ${ }^{10}$ Theoretical predictions have been made for ultrathin ${ }^{11-15}$ and magnetically doped ${ }^{8,16-19}$ topological insulators. Though much attention has focused on the usual transport properties of $\mathrm{Bi}_{2} \mathrm{Se}_{3}$, the compound initially has been demonstrated to be a thermoelectric material with high figure of merit $(Z T)^{20,21}$ with applications in thermoelectric and infrared devices. ${ }^{22,23} \mathrm{Bi}_{2} \mathrm{Te}_{3}$ films have been mechanically exfoliated ${ }^{24}$ and atomically grown, ${ }^{25}$ and are likely to show enhanced thermoelectric properties, ${ }^{26}$ for example, for heat to electricity conversion and cooling devices.

High ZT materials are desirable for improving the efficiency of thermoelectric devices, where $Z T=S^{2} \sigma T / \kappa$, with the temperature $T$, the thermoelectric power $S$, the electrical conductivity $\sigma$, and the thermal conductivity $\kappa$ (electrons and phonons). A significant $Z T$ has been demonstrated for the surface states of $\mathrm{Bi}_{2} \mathrm{Se}_{3},{ }^{27}$ due to enhancement of the thermoelectric power factor $S^{2} \sigma$ by the electronic conduction in the edge states and reduction of $\kappa$ by suppression of the phonon contribution. We consider ultrathin $\mathrm{Bi}_{2} \mathrm{Se}_{3}$ films in the presence of an exchange field to discuss thermoelectric phenomena and derive analytical expressions for the Berry phase mediated magnetic moment and orbital magnetization, motivated by recent experiments, ${ }^{7}$ and address the thermal

a)udo.schwingenschlogl@kaust.edu.sa. Tel.: +966(0)544700080. conductivity and thermoelectric power, which are experimentally accessible at reasonable temperatures due to the tunable band gap. ${ }^{27}$ Berry phase mediated thermoelectric properties are attracting much interest because of their technological importance. They have been derived in detail in Refs. 28-30 using Mott relations ${ }^{31,32}$ and have been applied to graphene ${ }^{33}$ and topological insulators. ${ }^{34}$ In Ref. 34, the thermal and Peltier/Nernst conductivities have been discussed for magnetic topological insulators and the experiments in Refs. 36-38 have shown good agreement with the Mott relations for temperatures up to $100 \mathrm{~K}$. Controlling the thermoelectric performance in topological insulator thin films by tuning the hybridization gap has been discussed in Ref. 35. Our results open experimental directions for thermoelectric transport experiments and device fabrication, since we establish for ultrathin $\mathrm{Bi}_{2} \mathrm{Se}_{3}$ the consequences of the strong spin orbit interaction and finite band gap that can be tuned by varying the thickness of the sample or by an exchange field, which leads to an enhancement of the thermoelectric power.

The surface Dirac fermions in $\mathrm{Bi}_{2} \mathrm{Se}_{3}$ obey the twodimensional Hamiltonian ${ }^{8}$

$$
H^{\tau_{z}}=v\left(p_{y} \sigma_{x}-\tau_{z} p_{x} \sigma_{y}\right)+\Delta \sigma_{z}+M \sigma_{z} \tau_{z},
$$

where we consider the topological insulator to be thin enough that there is a substantial overlap between the wave functions of the two surfaces giving rise to a hybridization $\Delta$. Moreover, $\tau_{z}= \pm 1$ represents the symmetric and antisymmetric combinations of the two surfaces, $M=g * \mu_{B} B / 2$ is the exchange (Zeeman) energy with $g *$ being the effective $g$-factor, $B$ is the exchange field along the $\mathrm{z}$-axis due to ferromagnetic ordering, $\left(\sigma_{x}, \sigma_{y}, \sigma_{z}\right)$ is the vector of Pauli matrices, and $v$ is the Fermi velocity of the Dirac fermions. Diagonalization of the Hamiltonian in Eq. (1) leads to the eigenvalues

$$
E_{t}^{\tau_{z}}=t \sqrt{(v \hbar k)^{2}+\left(\Delta+\tau_{z} M\right)^{2}}
$$

where $t= \pm 1$ represents the electrons and holes and $k=\sqrt{k_{x}^{2}+k_{y}^{2}}$. The corresponding eigenfunctions are 


$$
\begin{gathered}
\Psi_{t=+1}^{\tau_{z}}=\frac{1}{\sqrt{A}} \exp \left[i k_{x} x+i k_{y} y\right]\left(\begin{array}{c}
\tau_{z} i \cos \left[\theta_{\tau_{z}} / 2\right] e^{-\tau_{z} i \varphi} \\
\sin \left[\theta_{\tau_{z}} / 2\right]
\end{array}\right), \\
\Psi_{t=-1}^{\tau_{z}}=\frac{1}{\sqrt{A}} \exp \left[i k_{x} x+i k_{y} y\right]\left(\begin{array}{c}
i \sin \left[\theta_{\tau_{z}} / 2\right] e^{-\tau_{z} i \varphi} \\
-\tau_{z} \cos \left[\theta_{\tau_{z}} / 2\right]
\end{array}\right),
\end{gathered}
$$

where $A$ is the area of the system, $\cos \theta_{\tau_{z}}=\frac{\left(\Delta+\tau_{z} M\right)}{\sqrt{(v \hbar k)^{2}+\left(\Delta+\tau_{z} M\right)^{2}}}$, $\sin \theta_{\tau_{z}}=\frac{v \hbar k}{\sqrt{(v \hbar k)^{2}+\left(\Delta+\tau_{z} M\right)^{2}}}$, and $\varphi=\tan ^{-1} \frac{k_{y}}{k_{x}}$.

We show the eigenvalues given in Eq. (2) as a function of the dimensionless wave number in Fig. 1 for the symmetric (solid lines) and antisymmetric (dashed lines) combinations for $10 \mathrm{meV}$ exchange energy and hybridization energies of $0 \mathrm{meV}, 20 \mathrm{meV}, 35 \mathrm{meV}$, and $70 \mathrm{meV}$, corresponding to six quantiple layers (QL), $5 \mathrm{QL}, 4 \mathrm{QL}$, and 3 QL, respectively. ${ }^{3}$ We set $v=0.5 \times 10^{6} \mathrm{~m} / \mathrm{s}$. We find a well resolved band gap between the valence and conduction bands and notice that the surfaces are nondegenerate if $\Delta \neq 0$ and $M \neq 0$. In Fig. 2, we fix the hybridization energy $(20 \mathrm{meV}$ corresponding to a $5 \mathrm{~nm}$ thin film) and vary the exchange energy $(0 \mathrm{meV}, 10 \mathrm{meV}, 20 \mathrm{meV}$, and $40 \mathrm{meV})$, setting $g^{*}=39$, as observed for $\mathrm{Bi}_{2} \mathrm{Se}_{3} .{ }^{39}$ When the exchange and hybridization energies are equal, the gap closes for the antisymmetric combination and increases for the symmetric combination.
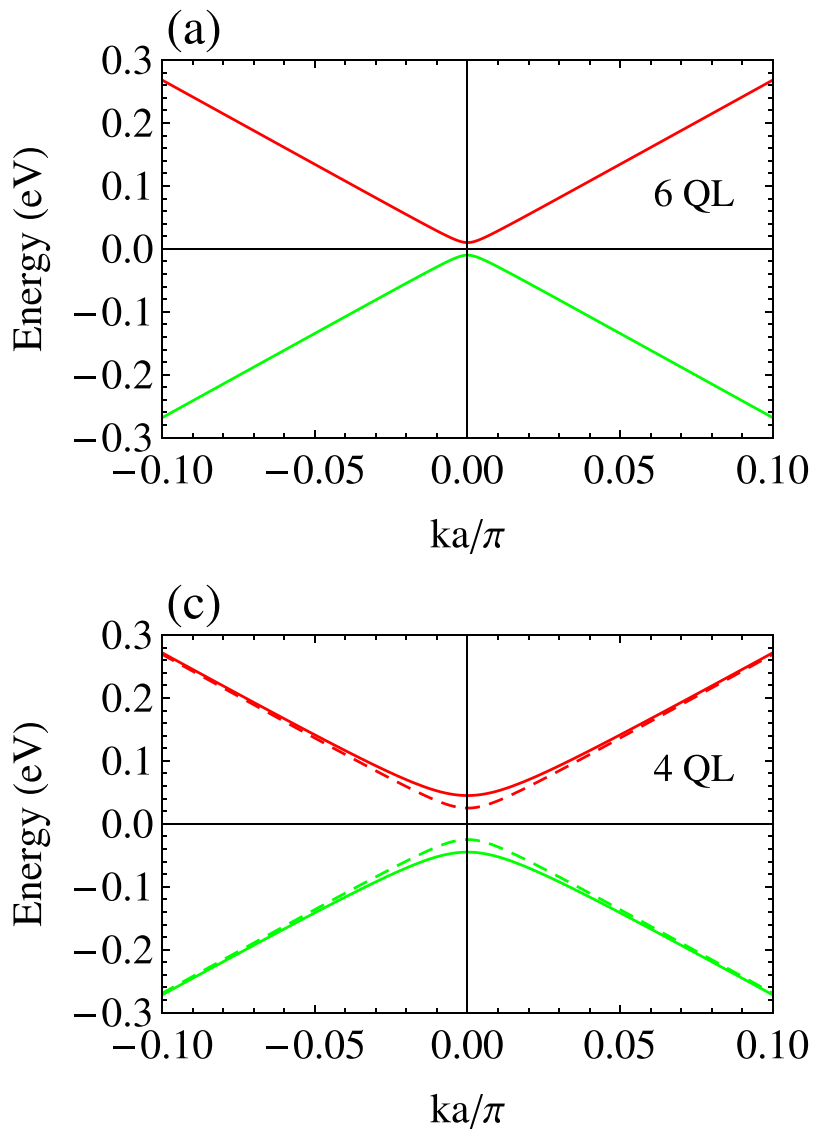

\section{RESULTS AND DISCUSSION}

The Berry phase is the phase acquired in a cyclic adiabatic process. Berry phase mediated orbital magnetism of Bloch electrons is generating interest in condensed matter physics due to its key role in transport phenomena. Besides the agreement with models based on the Mott relation with experiments, further advantages of this formulation are the analytical results that can be used to propose future experimental directions. In the definitions of the thermal conductivity

$$
\kappa_{i j}=\frac{\pi^{2} k_{B}^{2} T}{3 e^{2}} \sigma_{i j},
$$

Peltier/Nernst conductivity

$$
\alpha_{i j}=-\frac{\pi^{2} k_{B}^{2} T}{3 e} \frac{d \sigma_{i j}}{d E_{F}},
$$

and thermoelectric power

$$
S_{i j}=-\frac{\pi^{2} k_{B}^{2} T}{3 e} \frac{d}{d E_{F}} \ln \sigma_{i j},
$$

the Boltzmann constant amounts to $k_{B}=8.62 \times 10^{-5} \mathrm{eV} / \mathrm{K}$ and the Hall conductivity can be obtained from the standard Kubo formalism as ${ }^{28-30}$
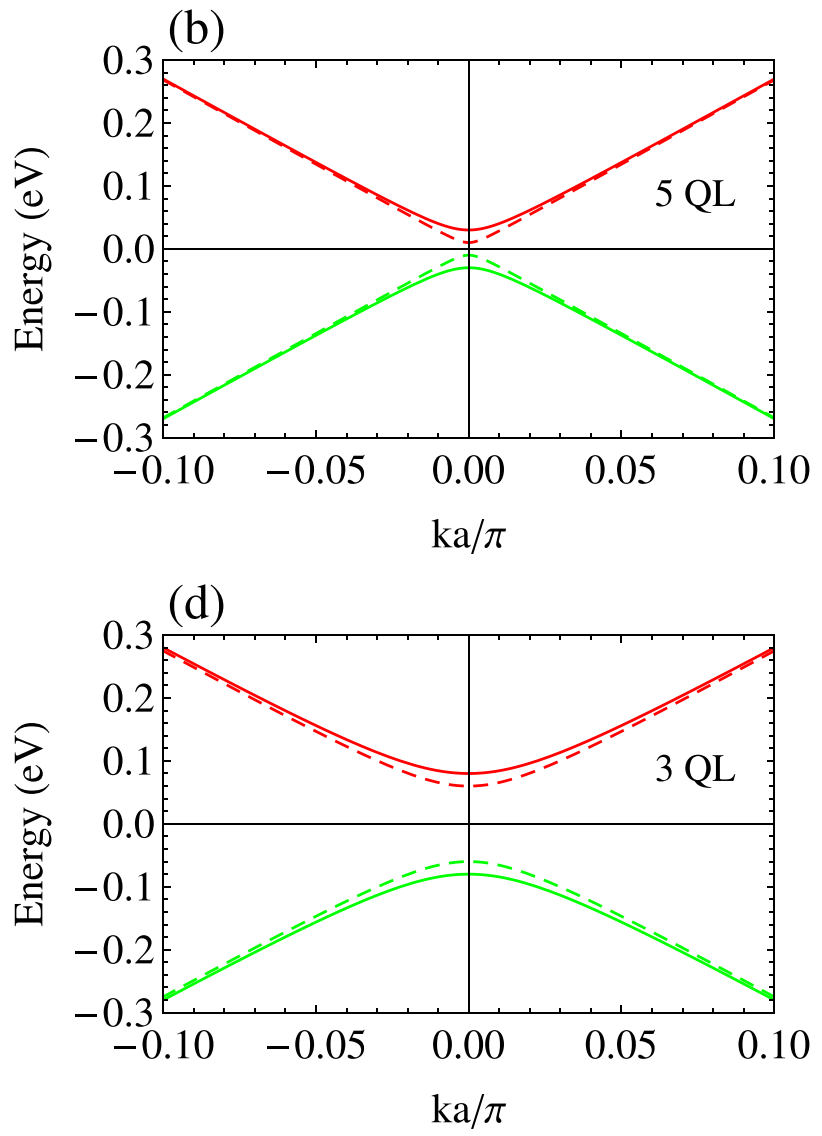

FIG. 1. Energy as a function of the dimensionless wave number for $10 \mathrm{meV}$ exchange energy and hybridization energies of (a) $0 \mathrm{meV}$ for $6 \mathrm{QL}$, (b) $20 \mathrm{meV}$ for 5 $\mathrm{QL}$, (c) $35 \mathrm{meV}$ for $4 \mathrm{QL}$, and (d) $70 \mathrm{meV}$ for $3 \mathrm{QL}$. Solid and dashed lines correspond to the symmetric and antisymmetric states, respectively. Red and green colors represent the conduction and valence bands, respectively. 
(a)

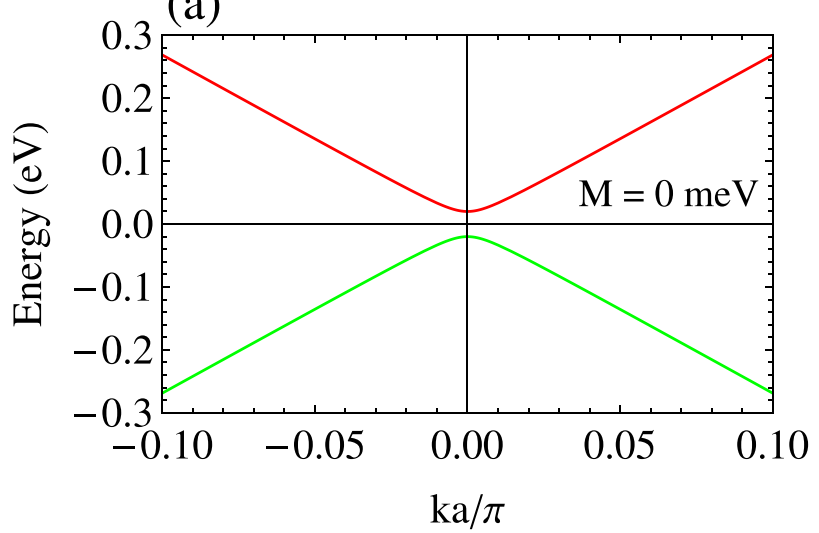

(c)

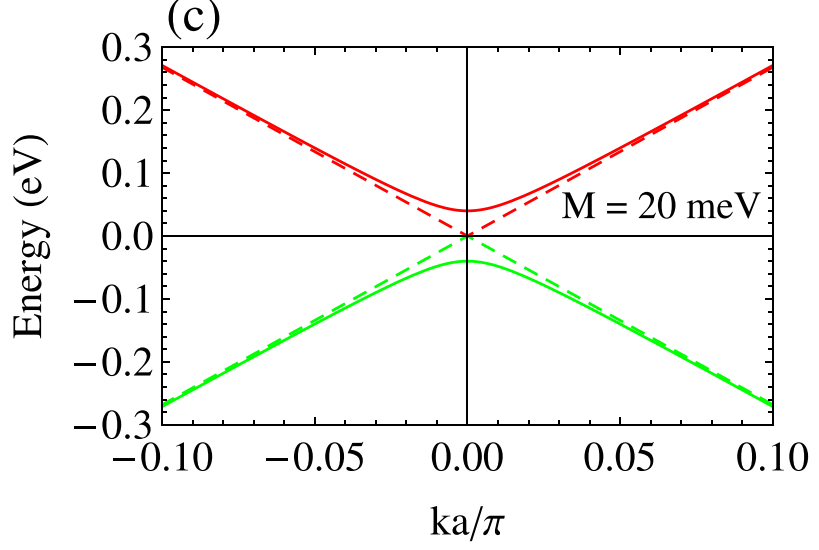

(b)

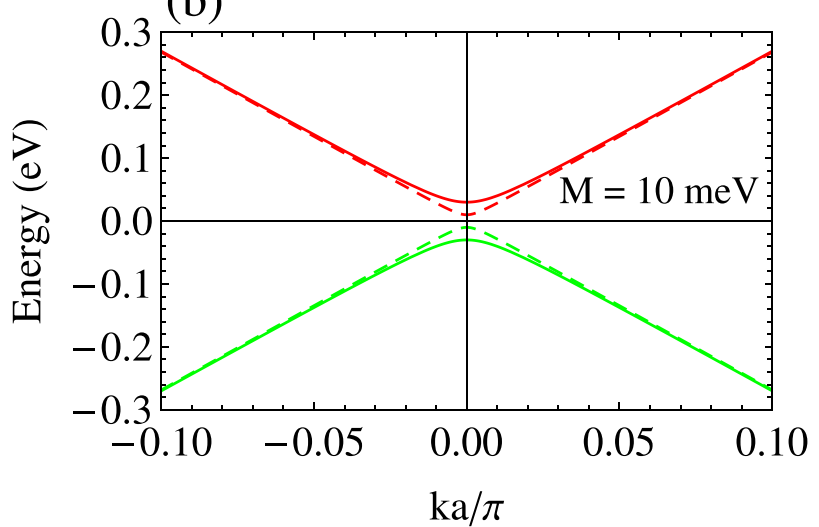

(d)

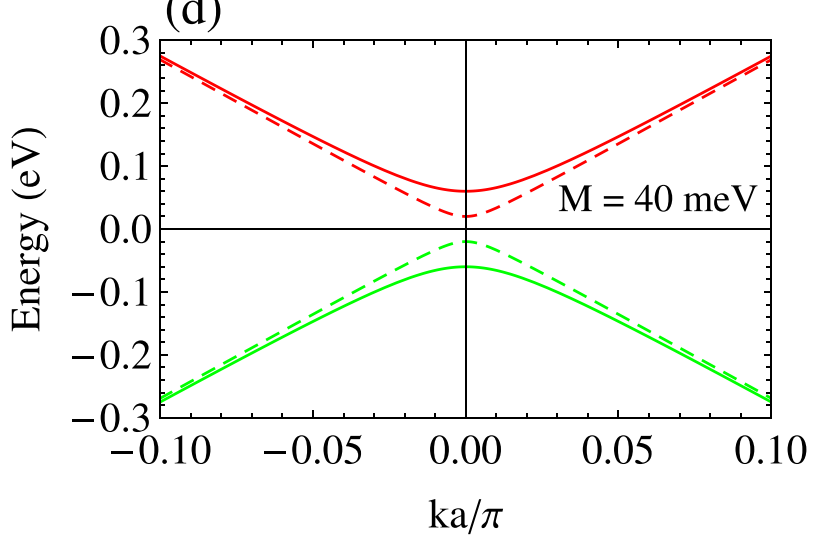

FIG. 2. Energy as a function of the dimensionless wave number for $20 \mathrm{meV}$ hybridization energy and exchange energies of (a) 0 meV, (b) $10 \mathrm{meV}$, (c) $20 \mathrm{meV}$, and (d) $40 \mathrm{meV}$. Solid and dashed lines correspond to the symmetric and antisymmetric states, respectively. Red and green colors represent the conduction and valence bands, respectively.

$$
\sigma_{i j}=\frac{e^{2}}{\hbar} \sum_{\tau_{z}, k} \Omega_{i j}^{\tau_{z}}(k)\left[f\left(E_{+}^{\tau_{z}}\right)-f\left(E_{-}^{\tau_{z}}\right)\right]
$$

where $f(E)$ is the Fermi-Dirac distribution function. The Berry curvature is given by

$$
\Omega_{i j}^{\tau_{z}}(k)=2 \operatorname{Im}\left[\left\langle\Psi_{+}^{\tau_{z}}\left|\hbar v_{i}\right| \Psi_{-}^{\tau_{z}}\right\rangle\left\langle\Psi_{-}^{\tau_{z}}\left|\hbar v_{j}\right| \Psi_{+}^{\tau_{z}}\right\rangle\right] /\left(E_{+}^{\tau_{z}}-E_{-}^{\tau_{z}}\right)^{2} .
$$

In a first step, we focus on the non-diagonal $(i=x, j=y)$ components of $\sigma_{i j}$. The velocity components $v_{x}=-\tau_{z} v \sigma_{y}$ and $v_{y}=v \sigma_{x}$ can be obtained from the Hamiltonian in Eq. (1). Using Eqs. (2)-(4), the expectation values of the velocities are derived and used in Eq. (9) to obtain the Berry curvature

$$
\Omega_{x y}^{\tau_{z}}(k)=\frac{-\tau_{z} \hbar^{2} v^{2}}{2} \frac{\Delta+\tau_{z} M}{\left[(v \hbar k)^{2}+\left(\Delta+\tau_{z} M\right)^{2}\right]^{3 / 2}}
$$

and the corresponding magnetic moment $m(k)=\frac{e}{\hbar} E_{t}^{\tau_{z}} \Omega_{x y}^{\tau_{z}}(k)$. For finite $\Delta$ or $M$, the magnetic moment has a peak at $k=0$. The moment of the surface state that we obtain for an exchange energy of $10 \mathrm{meV}$ is $35 \mathrm{Bohr}$ magnetons and may be varied by alteration of the exchange energy and/or thickness of the topological insulator. The magnetic moment turns out to be inversely proportional to the band gap. In agreement with our results, a higher moment than the usual spin magnetic moment has been demonstrated for a chemical potential difference between the two valleys in graphene. ${ }^{40,41}$ The corresponding orbital magnetization is obtained as ${ }^{28,30}$

$$
M\left(E_{F}\right)=\frac{e}{\hbar} \int \frac{d^{2} k}{(2 \pi)^{2}} E_{F} \Omega_{x y}^{\tau_{z}}(k)
$$

where $E_{F}$ is the Fermi energy. The Berry curvature and magnetization obtained by Eqs. (10) and (11) can be compared with the experimental results in Ref. 7. When $E_{F}^{+1}=E_{F}^{-1}$, the integral in Eq. (11) vanishes since the Berry curvature has opposite values on the two surfaces, $\Omega_{x y}^{+1}(k)=-\Omega_{x y}^{-1}(k)$. The orbital magnetization in the presence of a population difference $\left(E_{F}^{+1} \neq E_{F}^{-1}\right)$ has different values on the two surfaces, which results in a net magnetization of $\delta M=$ $2 / h\left(E_{F}^{+1}-E_{F}^{-1}\right)$ for $E_{F}>\Delta$ and $M$. This situation is analogous to the spin Hall effect ${ }^{42}$ and valley Hall effect ${ }^{40,41}$ in graphene. Our results are consistent with the experiments in Ref. 7 for finite $M(\Delta=0)$, because the magnetization obtained in Eq. (11) grows linearly with $E_{F}$, reflecting the relationship $d M\left(E_{F}\right) / d E_{F}=-\sigma_{x y} / e^{30}$ Using Eq. (10) in Eq. (8) we arrive in the case that $E_{F}$ lies in the conduction band at the Hall conductivity

$$
\sigma_{x y}=\frac{\tau_{z} e^{2}}{2 h} \frac{\Delta+\tau_{z} M}{\sqrt{\left(v \hbar k_{F}\right)^{2}+\left(\Delta+\tau_{z} M\right)^{2}}} .
$$


For symmetry reasons, an analogous result is obtained when $E_{F}$ lies in the valence band.

We now turn to the real part of the diagonal components $(i=j=x)$ of $\sigma_{i j}^{43}$ and calculate the longitudinal conductivity by the Kubo formula and a perturbative expansion within the first Born approximation, taking an ensemble average of uniform delta scatterers (potential $\left.V_{0}\right)$. We use the Streda ${ }^{44}$ version of the Kubo formula ${ }^{45}$

$$
\sigma_{i i}=-\frac{e^{2} \hbar}{4 \pi} \int d E \frac{\partial f}{\partial E} \operatorname{Tr}\left[v_{i}\left(G^{R}-G^{A}\right) v_{i} G^{A}-v_{i} G^{R} v_{i}\left(G^{R}-G^{A}\right)\right] .
$$

The unperturbed Green's function is given by $G_{0}^{R}$ $=[E-H+i 0]^{-1}$. Using the Hamiltonian $H=s_{z} v\left(\sigma_{x} p_{y}-\right.$ $\left.\sigma_{y} p_{x}\right)+\Delta s_{x}+M \sigma_{z}$ in $4 \times 4$ representation, ${ }^{8}$ where $\left(s_{x}, s_{y}, s_{z}\right)$ denotes the layer pseudospin, we obtain for the electronic contribution

$$
G_{0}^{R}=\sum_{s= \pm 1} \frac{1}{4\left(E-E_{s}+i 0\right)}\left[1+s_{x} s \sigma_{z}+\frac{\Delta+s M}{E_{s}}\left(s_{x}+s \sigma_{z}\right)+\frac{v \hbar\left(\sigma_{x} k_{y}-\sigma_{y} k_{x}\right)}{E_{s}} s_{z}+\frac{v \hbar\left(\sigma_{x} k_{x}+\sigma_{y} k_{y}\right)}{E_{s}} s_{y} s\right]
$$

with $E_{s}=\sqrt{(v \hbar k)^{2}+(\Delta+s M)^{2}}$. The perturbed Green's function is defined as $G^{R}=1 /\left[\left(G_{0}^{R}\right)^{-1}-\Sigma_{R}\right]$. For short range randomly distributed impurities, the self-energy is given by

$$
\Sigma_{R}=N V_{0}^{2} \int \frac{k d k d \phi}{(2 \pi)^{2}} G_{0}^{R} \approx-i N V_{0}^{2} E_{F} / 2 \hbar^{2} v^{2},
$$

where $N$ is the impurity concentration and we assume $E_{F} \gg$ $\Delta$ and $M$. This yields

$$
G^{R / A}=\sum_{s= \pm} \frac{1}{4\left(E \pm i \Gamma-E_{s}\right)}\left[1+s_{x} s \sigma_{z}+\frac{\Delta+s M}{E_{s}}\left(s_{x}+s \sigma_{z}\right)+\frac{v \hbar\left(\sigma_{x} k_{y}-\sigma_{y} k_{x}\right)}{E_{s}} s_{z}+\frac{v \hbar\left(\sigma_{x} k_{x}+\sigma_{y} k_{y}\right)}{E_{s}} s_{y} s\right],
$$

where $\Gamma=-\operatorname{Im} \Sigma_{R}$ is the broadening due to the finite quasiparticle life time $\tau$. Similar results are obtained for the hole contribution due to symmetry reasons.

Using Eq. (16) in Eq. (13) leads to the longitudinal conductivity (in the limit of zero temperature with chemical potential $E_{F}$ )

$$
\sigma_{x x}=\frac{e^{2}}{2 h} \frac{\tau E_{F}}{\hbar}\left(1-\frac{(\Delta+s M)^{2}}{E_{F}^{2}}\right),
$$

where the dependence on the hybridization and exchange energy is consistent with the band structure in Fig. 1. From $\sigma_{x x}$ we obtain

$$
S_{x x}=-\frac{\pi^{2} k_{B}^{2} T}{3 e E_{F}}\left(\frac{E_{F}^{2}+(\Delta+s M)^{2}}{E_{F}^{2}-(\Delta+s M)^{2}}\right)
$$

and

$$
k_{x x}=\frac{\pi^{2} k_{B}^{2} T}{3 e} \sigma_{x x} .
$$

The efficiency of a thermoelectric material is given by the figure of merit, which is derived from Eqs. (17)-(19) as

$$
Z T=\frac{\pi^{2} k_{B}^{2} T^{2}}{3 e E_{F}}\left(\frac{E_{F}^{2}+(\Delta+s M)^{2}}{E_{F}^{2}-(\Delta+s M)^{2}}\right)^{2}
$$

and thus depends on both $\Delta$ and $M$, being inversely proportional to the band gap. Analytical examination of Eq. (18) proves an enhanced thermoelectric power as compared with bulk $\mathrm{Bi}_{2} \mathrm{Se}_{3}$ due to the band gap of the surface states. It has been experimentally demonstrated that the gate voltage $\left(E_{F}\right.$; carrier concentration) dependence of the thermoelectric transport can be tuned by controlling the band gap. ${ }^{21} \mathrm{We}$ show numerical results for the thermoelectric power in Fig. 3 for $M=0$ and varying thickness of the sample. The maxima are more pronounced for larger band gaps, i.e., thinner samples, because a reduced film thickness enhances the thermoelectric power, consistent with experiments on ultrathin $\mathrm{Bi}_{2} \mathrm{Se}_{3} .{ }^{27}$

For the thermoelectric power as a function of $E_{F}$ for varying exchange energy and $\Delta=0(6 \mathrm{QL})$, we obtain similar results as shown in Fig. 3, where the magnitude again depends on the band gap. The values obtained for the cases demonstrated in Fig. 2 are an order of magnitude higher than observed for bulk $\mathrm{Bi}_{2} \mathrm{Se}_{3}$. An enhanced thermoelectric transport, on the other hand, has been demonstrated for Mn-doped $\mathrm{Bi}_{2} \mathrm{Se}_{3}{ }^{46}$ and Se-doped $\mathrm{Bi}_{2} \mathrm{Se}_{3},{ }^{47}$ consistent with our results. For a combination of magnetic doping and an ultrathin geometry, we obtain again similar curves as shown in Fig. 3, since both modifications affect the thermoelectric power in a similar way. The thermoelectric power as a function of temperature is shown in the top panel of Fig. 3 for the same parameters as used before, demonstrating the expected linear dependence. Finally, the thermoelectric efficiency is addressed in terms of the figure of merit in the bottom panel of Fig. 3. Clearly, a narrow band gap combined with a high mobility of the charge carriers is best suited for thermoelectric applications. While $\mathrm{Bi}_{2} \mathrm{Se}_{3}$ has a band gap of about 

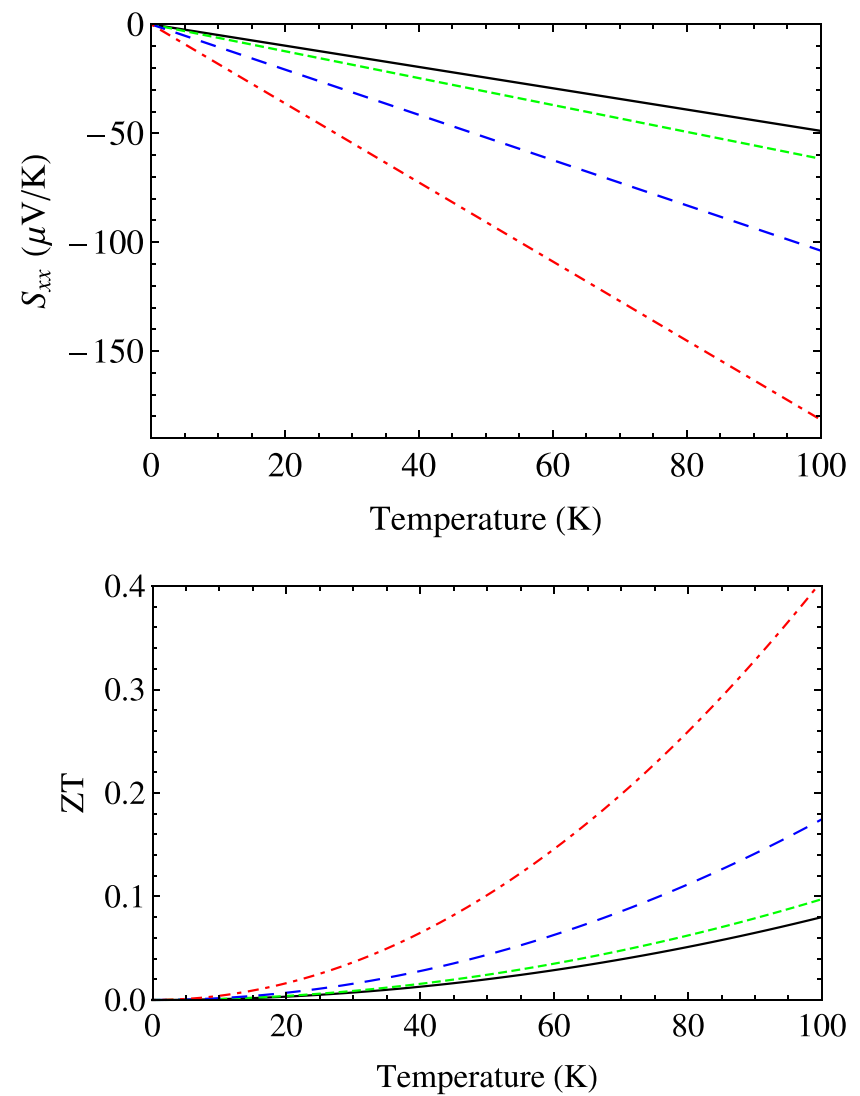

FIG. 3. Top: Thermoelectric power as a function of the temperature for $M=0$ and hybridization energies of (black) $20 \mathrm{meV}$ for $5 \mathrm{QL}$, (green) $35 \mathrm{meV}$ for $4 \mathrm{QL}$, (blue) $70 \mathrm{meV}$ for $3 \mathrm{QL}$, and (red) $126 \mathrm{meV}$ for $2 \mathrm{QL}$. Bottom: Figure of merit as a function of the temperature. We use $E_{F}=58 \mathrm{meV}$ for $\mathrm{Bi}_{2} \mathrm{Se}_{3}$ as obtained from Ref. 39 .

$300 \mathrm{meV}$ in the bulk and thus a small ZT, our results show that the latter is enhanced by an order of magnitude for ultrathin films, for example, due to the tunable band gap of the surface states.

\section{CONCLUSION}

To conclude, we have carried out analytical calculations for the thermoelectric transport in topological insulators, such as $\mathrm{Bi}_{2} \mathrm{Se}_{3}$ and $\mathrm{Bi}_{2} \mathrm{Te}_{3}$, and we have shown that a reduction of the sample thickness down to $2 \mathrm{~nm}$ enhances the band gap and therefore modifies the thermoelectric transport. Tunability of the band gap can be achieved by means of the exchange energy for magnetically doped topological insulators. The band gap can even reach zero (semiconductor to metal transition) by combination with the hybridization gap in ultrathin samples due to the interaction between the top and bottom surface sates. These results are intriguing for applications as the thermoelectric power is enhanced with respect to bulk samples and the transport properties become tunable.

\section{ACKNOWLEDGMENTS}

Research reported in this publication was supported by the King Abdullah University of Science and Technology (KAUST).
${ }^{1}$ H. Zhang, C. X. Liu, X. L. Qi, X. Dai, Z. Fang, and S. C. Zhang, Nat. Phys. 5, 438 (2009).

${ }^{2}$ Y. Xia, D. Qian, D. Hsieh, L. Wray, A. Pal, H. Lin, A. Bansil, D. Grauer, Y. S. Hor, R. J. Cava, and M. Z. Hasan, Nat. Phys. 5, 398 (2009).

${ }^{3}$ Y. Zhang, K. He, C. Z. Chang, C. L. Song, L. L. Wang, X. Chen, J. F. Jia, Z. Fang, X. Dai, W. Y. Shan, S. Q. Shen, Q. Niu, X. L. Qi, S. C. Zhang, X. C. Ma, and Q. K. Xue, Nat. Phys. 6, 584 (2010).

${ }^{4}$ M. Z. Hassan and C. L. Kane, Rev. Mod. Phys. 82, 3045 (2010).

${ }^{5}$ X. L. Qi and S. C. Zhang, Rev. Mod. Phys. 83, 1057 (2011).

${ }^{6}$ Y. L. Chen, J. H. Chu, J. G. Analytis, Z. K. Liu, K. Igarashi, H. H. Kuo, X. L. Qi, S. K. Mo, R. G. Moore, D. H. Lu, M. Hashimoto, T. Sasgawa, S. C. Zhang, I. R. Fisher, Z. Hussain, and Z. X. Shen, Science 329, 659 (2010).

${ }^{7}$ S. Y. Xu, M. Neupane, C. Liu, D. Zhang, A. Richardella, L. A. Wray, N. Alidoust, M. Leandersson, T. Balasubramanian, J. S. Barriga, O. Rader, G. Landolt, B. Slomski, J. H. Dil, J. Osterwalder, T. R. Chang, H. T. Jeng, H. Lin, A. Bansil, N. Samarth, and M. Z. Hasan, Nat. Phys. 8, 616 (2012).

${ }^{8}$ R. Yu, W. Zhang, H. J. Zhang, S. C. Zhang, X. Dai, and Z. Fang, Science 329, 61 (2010).

${ }^{9}$ P. Cheng, C. L. Song, T. Zhang, Y. Y. Zhang, Y. L. Wang, J. F. Jia, J. Wang, Y. Y. Wang, B. F. Zhu, X. Chen, X. Ma, K. He, L. L. Wang, X. Dai, Z. Fang, X. C. Xie, X. L. Qi, C. X. Liu, S. C. Zhang, and Q. K. Xue, Phys. Rev. Lett. 105, 076801 (2010).

${ }^{10}$ S. Cho, N. P. Butch, J. Paglione, and M. S. Fuhrer, Nano Lett. 11, 1925 (2011).

${ }^{11}$ J. Linder, T. Yokoyama, and A. Sudbø, Phys. Rev. B 80, 205401 (2009).

${ }^{12}$ C. X. Liu, H. J. Zhang, B. Yan, X. L. Qi, T. Frauenheim, X. Dai, Z. Fang, and S. C. Zhang, Phys. Rev. B 81, 041307 (2010).

${ }^{13}$ W. Y. Shan, H. Z. Lu, and S. Q. Shen, New J. Phys. 12, 043048 (2010).

${ }^{14}$ H.-Z. Lu, W.-Y. Shan, W. Yao, Q. Niu, and S. Q. Shen, Phys. Rev. B 81, 115407 (2010).

${ }^{15}$ H. Li, L. Sheng, D. N. Sheng, and D. Y. Xing, Phys. Rev. B 82, 165104 (2010).

${ }^{16}$ X. L. Qi, T. L. Hughes, and S. C. Zhang, Phys. Rev. B 78, 195424 (2008).

${ }^{17}$ Q. Meng, S. Vishveshwara, and T. L. Hughes, Phys. Rev. Lett. 109, 176803 (2012).

${ }^{18}$ J. Henk, M. Flieger, I. V. Maznichenko, I. Mertig, A. Ernst, S. V. Eremeev, and E. V. Chulkov, Phys. Rev. Lett. 109, 076801 (2012).

${ }^{19}$ F. Zhang, C. L. Kane, and E. J. Mele, Phys. Rev. Lett. 110, 046404 (2013).

${ }^{20} \mathrm{~S}$. Scherrer and H. Scherrer, "Bismuth terruride, antimony telluride, and their solid solutions," in CRC Handbook of Thermoelectrics, edited by D. M. Rowe (CRC, Boca Raton, FL, 1995), Chap. 19.

${ }^{21}$ G. J. Snyder and E. S. Toberer, Nature Mater. 7, 105 (2008).

${ }^{22}$ D. Arivuoli, F. D. Gnanam, and P. Ramasamy, J. Mater. Sci. Lett. 7, 711 (1988).

${ }^{23}$ H. T. El-Shair, A. M. Ibrahim, E. A. El-Wahabb, M. A. Afify, and F. A. El-Salam, Vacuum 42, 911 (1991).

${ }^{24}$ D. Teweldebrhan, V. Goyal, and A. A. Balandin, Nano Lett. 10, 1209 (2010).

${ }^{25}$ Y. Y. Li, G. Wang, X. G. Zhu, M. H. Liu, C. Ye, X. Chen, Y. Y. Wang, K. He, L. L. Wang, X. C. Ma, H. J. Zhang, X. Dai, Z. Fang, X. C. Xie, Y. Liu, X. L. Qi, J. F. Jia, S. C. Zhang, and Q. K. Xue, Adv. Mater. 22, 4002 (2010).

${ }^{26}$ J. Maassen and M. Lundstrom, Appl. Phys. Lett. 102, 093103 (2013).

${ }^{27}$ Y. Sun, H. Cheng, S. Gao, Q. Liu, Z. Sun, C. Xiao, C. Wu, S. Wei, and Y. Xie, J. Am. Chem. Soc. 134, 20294 (2012).

${ }^{28}$ D. Xiao, M. C. Chang, and Q. Niu, Rev. Mod. Phys. 82, 1959 (2010).

${ }^{29}$ D. Xiao, Y. Yao, Z. Fang, and Q. Niu, Phys. Rev. Lett. 97, 026603 (2006).

${ }^{30}$ Z. Wang, P. Zhang, and J. Shi, Phys. Rev. B 76, 094406 (2007).

${ }^{31}$ M. Cutler and N. F. Mott, Phys. Rev. 181, 1336 (1969).

${ }^{32}$ C. Lee, G. Yi, Y. M. Zuev, and P. Kim, Appl. Phys. Lett. 94, 022106 (2009).

${ }^{33}$ C. Zhang, S. Tewari, and S. D. Sarma, Phys. Rev. B 79, 245424 (2009).

${ }^{34}$ T. Yokoyama and S. Murakami, Phys. Rev. B 83, 161407(R) (2011).

${ }^{35}$ P. Ghaemi, R. S. K. Mong, and J. E. Moore, Phys. Rev. Lett. 105, 166603 (2010).

${ }^{36}$ P. Wei, W. Bao, Y. Pu, C. N. Lau, and J. Shi, Phys. Rev. Lett. 102, 166808 (2009).

${ }^{37}$ D. Wang and J. Shi, Phys. Rev. B 83, 113403 (2011), and references therein. 
${ }^{38}$ C.-R. Wang, W.-S. Lu, L. Hao, W.-L. Lee, T.-K. Lee, F. Lin, I.-C. Cheng, and J.-Z. Chen, Phys. Rev. Lett. 107, 186602 (2011).

${ }^{39}$ A. A. Taskin and Y. Ando, Phys. Rev. B 84, 035301 (2011).

${ }^{40}$ D. Xiao, W. Yao, and Q. Niu, Phys. Rev. Lett. 99, 236809 (2007).

${ }^{41}$ A. Rycerz, J. Tworzydlo, and C. W. J. Beenakker, Nat. Phys. 3, 172 (2007).

${ }^{42}$ S. Murakami, N. Nagaosa, and S. C. Zhang, Science 301, 1348 (2003).

${ }^{43}$ K. Nomura and A. H. MacDonald, Phys. Rev. Lett. 98, 076602 (2007).
${ }^{44}$ P. Streda, J. Phys. C 15, L717 (1982).

${ }^{45}$ N. A. Sinitsyn, J. E. Hill, H. Min, J. Sinova, and A. H. MacDonald, Phys. Rev. Lett. 97, 106804 (2006).

${ }^{46}$ Y. H. Choi, N. H. Jo, K. J. Lee, H. W. Lee, Y. H. Jo, J. Kajino, T. Takabatake, K. T. Ko, J. H. Park, and M. H. Jung, Appl. Phys. Lett. 101, 152103 (2012).

${ }^{47}$ A. Soni, Z. Yanyuan, Y. Ligen, M. K. K. Aik, M. S. Dresselhaus, and Q. Xiong, Nano Lett. 12, 1203 (2012). 